\section{BMJ Open Respiratory Research}

\title{
Monitoring tuberculosis contact tracing outcomes in Western Sydney, Australia
}

\author{
Laila Parvaresh, ${ }^{1}$ Shopna K Bag, ${ }^{1,2}$ Jin-Gun Cho, ${ }^{2,3,4}$ Neil Heron, ${ }^{4}$ Hassan Assareh, ${ }^{5}$ \\ Sophie Norton, ${ }^{1}$ Stephen Corbett, ${ }^{1,2}$ Ben J Marais ${ }^{2,6}$
}

To cite: Parvaresh L, Bag SK, Cho J-G, et al. Monitoring tuberculosis contact tracing outcomes in Western Sydney, Australia. BMJ Open Resp Res 2018;5:e000341. doi:10.1136/ bmjresp-2018-000341

Received 27 July 2018 Revised 26 August 2018 Accepted 28 August 2018

\section{Check for updates}

(c) Author(s) (or their employer(s)) 2018. Re-use permitted under CC BY-NC. No commercial re-use. See rights and permissions. Published by BMJ.

${ }^{1}$ Public Health Unit, Western Sydney Local Health District, North Parramatta, New South Wales, Australia

${ }^{2}$ The University of Sydney, Sydney, New South Wales, Australia

${ }^{3}$ Respiratory and Sleep Medicine, Westmead Hospital, Westmead, New South Wales, Australia

${ }^{4}$ Parramatta Chest Clinic, Parramatta, New South Wales Australia

${ }^{5}$ Epidemiology and Health Analytics, Western Sydney Local Health District, North Parramatta, New South Wales, Australia

${ }^{6}$ Department of Infectious Diseases and Microbiology, The Children Hospital at Westmead, Westmead, New South Wales, Australia

Correspondence to

Shopna K Bag;

shopna.bag@health.nsw. gov.au

\section{ABSTRACT}

Treatment of latent tuberculosis infection (LTBI) is an important component of strategies to achieve global tuberculosis (TB) elimination, but implementation is rarely monitored. This is a retrospective review of TB contact tracing outcomes at one of the busiest TB clinics in Australia, measured against the Centre for Disease Control and Prevention performance indicators. In total, 45 of 53 (85\%) pulmonary TB cases had 171 close contacts, of whom $139(81 \%)$ were evaluated with a tuberculin skin test (TST); 58 of 139 (42\%) were positive at baseline. Among 57 close contacts of 16 sputum smear-positive TB cases, the elicitation, evaluation, initiation of LTBI treatment and completion rates were $93 \%, 86 \%, 14 \%$ and $100 \%$, and among 114 close contacts of 37 sputum smearnegative pulmonary TB cases $81 \%, 83 \%, 16 \%$ and $89 \%$, respectively. Of 79 contacts with an initial negative TST, 19 of $47(40 \%)$ demonstrated TST conversion when retested; 5 of $19(26 \%)$ were offered LTBI treatment. Four secondary TB cases were identified. One incident TB case developed a pleural effusion 5 months after TST conversion, despite LTBI treatment. Apart from young children, LTBI treatment was inconsistently initiated in household TB contacts. Safe and pragmatic treatment options, as well as functional monitoring frameworks, are essential to improve LTBI treatment implementation.

\section{INTRODUCTION}

In May 2014, the World Health Assembly approved the new WHO End TB strategy, which aims to reduce tuberculosis (TB) incidence and TB-related mortality by $90 \%$ and $95 \%$, respectively, by $2035 .{ }^{1}$ For low TB incidence countries (defined as $<10$ TB cases per 100000 population), an action framework was developed with a strong emphasis on enhanced use of TB preventive therapy. ${ }^{2}$ Effectively interrupting TB transmission and reducing the pool of individuals with latent infection from whom future cases may emerge will be necessary to meet the End TB targets. ${ }^{3}$ The transmission chain can be interrupted if effective contact tracing identifies and treats all prevalent secondary cases, as well as those with latent tuberculosis infection (LTBI). ${ }^{23}$ Both the 'End TB Strategy' and the 'Low-incidence country action framework' highlight contact tracing and LTBI treatment as key steps towards ending the TB epidemic. ${ }^{24}$ In Australia, this principle has been advocated by the National Strategic Plan for Control of Tuberculosis 2011-2015, ${ }^{5}$ and Australia signed the 'Low-incidence country action framework' at the World TB Conference in Barcelona in October 2014. However, despite a low TB notification rate of $\sim 6$ per 100000 population per year (5.8 per 100000 population in 2013), ${ }^{6}$ progress towards TB elimination has been slow.

The state of New South Wales reports the highest TB case numbers in Australia, with most cases diagnosed in metropolitan Sydney. ${ }^{7}$ Within Sydney the Western Sydney Local Health District (LHD) has the highest TB incidence rate. ${ }^{8}$ Western Sydney LHD has a cosmopolitan population with migrants from many TB endemic countries, ${ }^{9} 10$ and is projected to have the highest population growth in New South Wales (2.3\% per year) in the coming years, mainly driven by migration from $\mathrm{TB}$ endemic countries. In view of the associated TB importation risk, it is important to establish robust public health systems that will prevent local transmission whenever an infectious TB case is identified. ${ }^{2}$ The Western Sydney LHD developed a TB control strategy aligned with key priorities identified in the 'Low-incidence countries action framework', which encourages the expanded use of preventive therapy. ${ }^{2}$ Here, we assess the performance of contact tracing services in Western Sydney and make recommendation in relation to service improvement and for ongoing quality assurance audits.

\section{METHODS}

We performed a retrospective review of all pulmonary TB cases diagnosed at Parramatta Chest Clinic during 2013 (1 January-31 December) and compared the outcomes achieved with relevant Centre for Disease Control and Prevention (CDC) performance indicators. 


\section{Study setting}

New South Wales identified 1386 confirmed TB cases from 2012 to 2014 ( 460 per annum), with an average annual incidence of 6.3 per 100000 population, 14.1 per 100000 in Western Sydney LHD. ${ }^{8}$ Close contacts of infectious TB cases are routinely screened for LTBI and offered a choice between LTBI treatment (usually with isoniazid for 6-9 months) or chest radiograph (CXR) surveillance, if considered to have been infected during the recent exposure. ${ }^{7}$ Close contact is defined as being a household contact or having spent prolonged periods of time in close proximity with a patient with infectious TB. CXR surveillance and clinical follow-up are usually undertaken at 3,6, 12 and 24 months, in the absence of symptoms of active disease. ${ }^{11} \mathrm{~TB}$ services in Western Sydney LHD, including contact tracing, are delivered by the Parramatta Chest Clinic with the support of local respiratory physicians, and for large screenings the Western Sydney Public Health Unit. ${ }^{12}$

Contact screening was conducted by performing a tuberculin skin test (TST) and CXR. The TST was performed by intradermal injection of $0.1 \mathrm{~mL}$ of purified protein derivative into the volar aspect of the forearm and measuring the diameter of induration (skin elevation, not erythema) in 2-3 days. ${ }^{13}$ A positive reaction was defined as $\geq 10 \mathrm{~mm}$, but the interpretation of TST results between 5 and $10 \mathrm{~mm}$ was variable, as some of the clinicians followed the CDC criteria of $\geq 5 \mathrm{~mm}$ in recent contacts ${ }^{13}$ and others followed the WHO criteria of $\geq 10 \mathrm{~mm}$ in child contacts who are HIV-uninfected. ${ }^{14}$ In general, if the TST reading was $5-10 \mathrm{~mm}$, it was considered as indeterminate and contacts were assigned to clinical follow-up. All cases with documented TST conversion had an initial negative TST that became positive on retesting $2-3$ months later. ${ }^{15}$

\section{Data sources and analysis}

TB case data were collected from the Notifiable Conditions Information Management System, which is a New South Wales secure public health notification database. ${ }^{16}$ Information on all contacts was collected from original TB contact tracing forms and a local Clinical Surveillance System database that recorded sociodemographic parameters, clinical history and case management plans. Sociodemographic and clinical variables for de-identified cases and contacts were collected and recorded on separate data collection sheets. Additional variables such as history of BCG vaccination and being under TB surveillance, often referred to as a $\mathrm{TB}$ undertaking in New South Wales, were also recorded.

Descriptive data analysis was performed using R V.3.2.0 (R Foundation for Statistical Computing, 2015, Vienna, Austria). Performance indicators were defined based on the CDC performance targets for 2020, which capture information on the contact screening cascade of care. The specific indicators were categorised as (1) contact elicitation, (2) contact evaluation, (3) LTBI treatment initiation and (4) LTBI treatment completion. ${ }^{17}$

\section{RESULTS}

During 2013, 107 TB cases were managed at Parramatta Chest Clinic; 53 had pulmonary TB, of whom 16 (30\%) were sputum smear-positive. Table 1 summarises the relevant characteristics of the pulmonary $\mathrm{TB}$ cases identified. Most (50 of 53; 94\%) were born overseas, and CXR abnormalities warranting active postmigration TB surveillance were identified at migration screening in 16 of $50(32 \%)$, of whom 7 of $16(44 \%)$ were diagnosed with pulmonary TB after the period of active surveillance. In total, $45(85 \%)$ pulmonary TB cases identified close contacts, with a median of 3 close contacts per case (IQR: 2-4). Of the 243 close contacts elicited, 171 (70\%) resided in Western Sydney LHD and were included in this analysis. The other 72 close contacts were referred to their local chest clinics for management.

Figure 1 reflects the data for the 171 close contacts residing in Western Sydney LHD; 144 (84\%) presented for TB screening, of whom $120(70 \%)$ received TST and CXR and $19(11 \%)$ only a TST. Five (3\%) contacts had a previous positive TST and received a CXR only. Nearly everyone who received a TST (137 of $139 ; 99 \%)$ returned for the reading; $54(39 \%)$ recorded a positive $(\geq 10 \mathrm{~mm})$, $4(3 \%)$ an intermediate $(5-9 \mathrm{~mm})$ and $79(58 \%)$ a negative $(<5 \mathrm{~mm})$ reading. Of the 79 contacts with a negative first TST, $49(62 \%)$ had a second TST, of whom 47 $(96 \%)$ returned for the reading; $19(40 \%)$ demonstrated TST conversion. Those with a negative or absent second TST reading were discharged from follow-up. Contacts with an indeterminate first TST reading (5-9 $\mathrm{mm}$ ) were assigned to CXR surveillance, which only 1 (25\%) completed. Among the close contacts identified, 14 of $171(8 \%)$ were young children $(<5$ years of age). None were diagnosed with $\mathrm{TB}$ disease, but two had a positive TST and completed LTBI treatment. One had a TST reading of $6 \mathrm{~mm}$ (no BCG vaccination; repeat TST 12 $\mathrm{mm}$ ) and was put on CXR surveillance, which was not completed. Of the 11 children with a negative first TST, none were provided 'window prophylaxis', six (60\%) had a second TST that was negative and all were discharged from follow-up.

Overall, 64 of $171(37 \%)$ close contacts completed screening and follow-up as advised, $3(2 \%)$ were transferred to other area health services, $27(16 \%)$ failed to present for screening, and 77 (45\%) initiated screening but did not complete their follow-up. We identified four secondary TB cases; three were diagnosed during initial screening (prevalent cases) and one during subsequent follow-up (incident case). Three of the secondary TB cases had negative cultures, but one was sputum smear-positive and culture-positive with a 24-locus multiple interspersed repetitive unit profile that was identical to the likely source case. The one incident case that developed TB after documented TST conversion did so while on LTBI treatment. He collected monthly isoniazid tablets for 5 months (adherence with daily unsupervised therapy uncertain) before developing a pleural effusion that was culture-negative but treated as TB. The organism isolated 
Table 1 Characteristics of pulmonary TB cases diagnosed at Parramatta Chest Clinic, Sydney, Australia (2013)

\begin{tabular}{|c|c|c|c|c|}
\hline \multirow[b]{2}{*}{ Characteristics of PTB cases } & \multicolumn{3}{|c|}{ PTB cases $(n=53)$ with close contacts } & \multirow[b]{2}{*}{$\begin{array}{l}\text { Contacts } \\
(n=171)\end{array}$} \\
\hline & $\begin{array}{l}\text { Any close contacts } \\
\mathrm{n}(\%)\end{array}$ & $\begin{array}{l}\text { No declared contacts } \\
\text { n (\%) }\end{array}$ & $\begin{array}{l}\text { Contacts }<5 \text { years } \\
\mathrm{n}(\%)\end{array}$ & \\
\hline \multicolumn{5}{|l|}{ Age group (years) } \\
\hline$<5$ & 0 & 0 & 0 & 0 \\
\hline $5-14$ & 0 & $1(2)$ & 0 & 0 \\
\hline $15-24$ & $6(11)$ & $1(2)$ & $2(4)$ & $46(27)$ \\
\hline $25-49$ & $21(40)$ & $4(7)$ & $5(9)$ & $59(34)$ \\
\hline$\geq 50$ & $18(34)$ & $2(4)$ & $4(8)$ & $66(39)$ \\
\hline \multicolumn{5}{|l|}{ Gender } \\
\hline Female & $19(36)$ & $5(9)$ & $6(11)$ & $87(51)$ \\
\hline Male & $26(49)$ & $3(6)$ & $5(9)$ & $84(49)$ \\
\hline \multicolumn{5}{|l|}{ Immigration status } \\
\hline Permanent visa & $34(64)$ & $5(9)$ & $7(13)$ & $138(81)$ \\
\hline Student visa & $5(9)$ & $2(4)$ & $1(2)$ & $14(8)$ \\
\hline Other & $6(11)$ & $1(2)$ & $3(6)$ & $19(11)$ \\
\hline \multicolumn{5}{|l|}{ PTB type } \\
\hline Sputum smear-positive & $15(28)$ & $1(2)$ & $3(6)$ & $57(33)$ \\
\hline Sputum smear-negative & $30(57)$ & $7(13)$ & $8(15)$ & $112(65)$ \\
\hline Culture-positive* & $33(62)$ & $5(9)$ & $9(17)$ & $140(82)$ \\
\hline Culture-negative & $11(21)$ & $2(4)$ & $2(4)$ & $29(17)$ \\
\hline \multicolumn{5}{|l|}{ Active TB surveillance $†$} \\
\hline Current & $4(8)$ & $5(9)$ & $1(2)$ & $10(6)$ \\
\hline Previous & $5(9)$ & $2(4)$ & $1(2)$ & $15(9)$ \\
\hline Never & $35(66)$ & $1(2)$ & $9(17)$ & $146(85)$ \\
\hline \multicolumn{5}{|l|}{ Cough at presentation $\ddagger$} \\
\hline Yes & $30(57)$ & $3(6)$ & $6(11)$ & $121(71)$ \\
\hline No & $14(26)$ & $5(9)$ & $5(9)$ & $49(29)$ \\
\hline
\end{tabular}

*No cultures performed or no data in two cases.

†Refers to people who are, or have previously been, on a so-called 'TB undertaking'. This involves active TB surveillance (for up to 2 years) after migration to Australia in people who had an abnormal chest radiograph at immigration screening, but no signs or symptoms suggestive of active TB.

$\ddagger$ No symptoms recorded at presentation in one case.

PTB, pulmonary tuberculosis; TB, tuberculosis.

from his likely source case was sensitive to isoniazid. He received a prolonged course of standard four-drug treatment and made a complete recovery.

Table 2 reflects the management of close contacts of sputum smear-positive and smear-negative pulmonary TB cases compared with the CDC performance targets. Of 16 sputum smear-positive cases, 15 (94\%) indicated at least one close contact. In total 57 close contacts were identified, of whom $49(86 \%)$ were evaluated. At baseline evaluation, 16 of 49 (33\%) were diagnosed with LTBI, of whom $1(6 \%)$ was started on LTBI treatment and the rest $(15 ; 94 \%)$ opted for CXR surveillance, which only 4 of $15(27 \%)$ completed. Of those who received a second TST, 5 of 21 (24\%) were diagnosed with TST conversion, of whom 2 received and completed LTBI treatment and 3 opted for CXR surveillance, which only $1(33 \%)$ completed. Among the close contacts of all sputum pulmonary TB cases (both sputum smear-positive and smear-negative), 58 of 137 (42.3\%) of those tested had a positive TST at baseline; 7 (12\%) commenced LTBI treatment and $51(88 \%)$ opted for CXR surveillance, which 15 (29\%) completed. Of those who received a second TST, 19 of $49(39 \%)$ were diagnosed with TST conversion, of whom $5(26 \%)$ received and completed LTBI treatment and $14(74 \%)$ were placed on CXR surveillance, which 3 (21\%) completed.

\section{DISCUSSION}

The performance of TB contact tracing activities is not included in routine WHO reports and infrequently monitored, even in low-incidence settings with adequate 


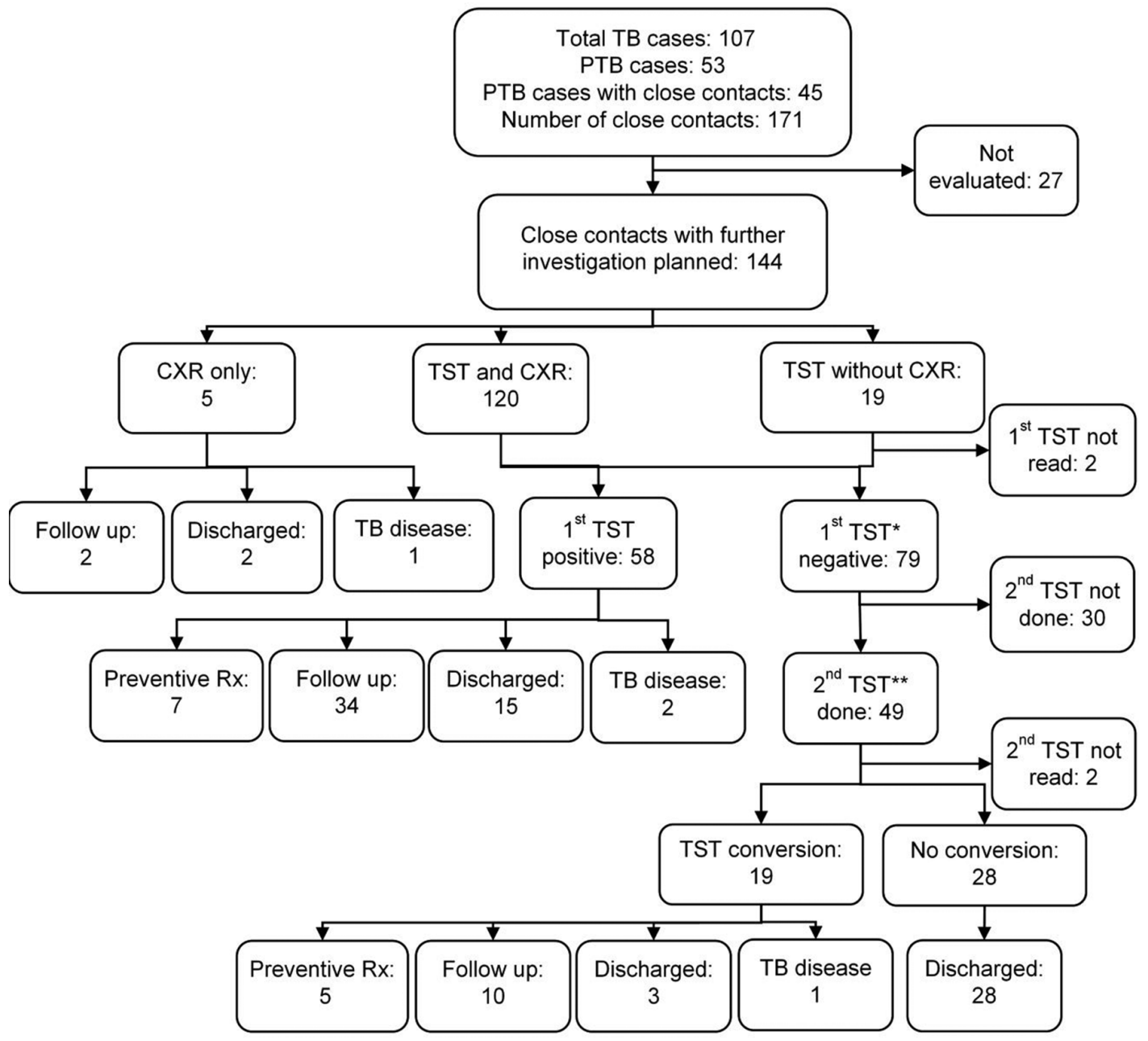

Figure 1 Flow diagram demonstrating the management of close contacts of pulmonary TB cases diagnosed at Parramatta Chest Clinic, Sydney, Australia. ${ }^{*}$ None of the contacts with a negative first TST had relevant CXR abnormalities. ${ }^{*} A$ second TST was done if the first TST was negative and performed within the theoretical 'conversion window'. CXR, chest radiograph; PTD, pulmonary tuberculosis; Rx, treatment; TB, tuberculosis; TST, tuberculin skin test.

resources. However, with a renewed emphasis on TB elimination in low-incidence settings, expectations are changing. In Parramatta Chest Clinic, the elicitation of close $\mathrm{TB}$ contacts and screening of these contacts for TB and LTBI were generally well done. Although assessment rates were similar to those reported in studies from the USA $(88 \%)^{18}$ and UK $(86 \%),{ }^{19}$ they still fall short of the CDC target of $93 \% .{ }^{17}$ A major implementation gap compared with the CDC targets was the fact that LTBI treatment was initiated in $<20 \%$ of those with documented LTBI. At the time, New South Wales guidance only encouraged LTBI treatment in those considered to be at increased risk of TB disease progression, such as young children and people with significant immune compromise, or those with documented recent TB infection (TST conversion)..$^{811}$

Few young children ( $<5$ years of age) had documented LTBI, but $100 \%$ of those with a TST reading $\geq 10 \mathrm{~mm}$ received preventive therapy and completed their full course of treatment. A single child with an indeterminate $(6 \mathrm{~mm})$ TST was placed on CXR surveillance. Although none of the children whose first TST was negative demonstrated TST conversion on repeat testing, "window prophylaxis' was not offered. 'Window prophylaxis' requires consideration in young children who are at high risk of rapid disease progression following primary $\mathrm{Myco-}$ bacterium tuberculosis infection, ${ }^{20}$ even if the initial TST or interferon- $\gamma$ release assay (IGRA) result performed during the 'conversion window' (first 6-8 weeks after the end of TB exposure) is negative. International guidance encourages the provision of preventive therapy in this vulnerable population irrespective of the TST result, particularly in settings where LTBI testing is problematic. ${ }^{314}$ 21-23 The most recent WHO LTBI treatment guidance encourages the use of water-dispersible isoniazid and rifampicin fixed-dose combination tablets for a period of 3 months. ${ }^{21}$ These 'child-friendly' medications are now available through the Global Drug Facility, ${ }^{24}$ also to high-income countries like Australia, but given incountry restrictions on the registration of therapeutic drugs the use of special access schemes is required to make these drugs available to children. 
Table 2 Management of close contacts of pulmonary TB cases compared with CDC performance targets ${ }^{\star}$

\begin{tabular}{|c|c|c|c|c|c|}
\hline \multirow[b]{2}{*}{ CDC indicator } & \multicolumn{4}{|c|}{ Age group of PTB cases and close contacts } & \multirow[b]{2}{*}{$\begin{array}{l}\text { CDC target* } \\
(\%)\end{array}$} \\
\hline & $\begin{array}{l}<5 \text { years } \\
\text { fraction }(\%)\end{array}$ & $\begin{array}{l}5-14 \text { years } \\
\text { fraction }(\%)\end{array}$ & $\begin{array}{l}\geq 15 \text { years } \\
\text { fraction }(\%)\end{array}$ & $\begin{array}{l}\text { Total fraction } \\
(\%)\end{array}$ & \\
\hline \multicolumn{6}{|l|}{ Sputum smear-positive PTB cases $(n=16)$} \\
\hline Cases in whom close contacts were elicited $\dagger$ & NA & NA & 15/16 (93) & $15 / 16(93)$ & 100 \\
\hline $\begin{array}{l}\text { Close contacts evaluated } \neq \\
\text { (TST and CXR, TST or CXR) }\end{array}$ & $5 / 5(100)$ & $6 / 9(66)$ & $38 / 43(91)$ & 49/57 (86) & 93 \\
\hline Preventive therapy initiated§ & $1 / 1(100)$ & $0 / 2(0)$ & $2 / 18(11)$ & $3 / 21(14)$ & 91 \\
\hline Preventive therapy completed & $1 / 1(100)$ & NA & $2 / 2(100)$ & $3 / 3(100)$ & 81 \\
\hline \multicolumn{6}{|l|}{ Sputum smear-negative PTB cases $(n=37)$} \\
\hline Cases in whom close contacts were elicited $\dagger$ & NA & NA & $30 / 37(81)$ & $30 / 37(81)$ & 100 \\
\hline $\begin{array}{l}\text { Close contacts evaluated } \neq \\
\text { (TST and CXR, TST or CXR) }\end{array}$ & $9 / 9(100)$ & $11 / 13(85)$ & 75/92 (82) & $95 / 114(83)$ & 93 \\
\hline Preventive therapy initiated§ & $1 / 2(50)$ & 2/7 (29) & $6 / 47(13)$ & $9 / 56(16)$ & 91 \\
\hline Preventive therapy completed & $1 / 1(100)$ & 2/2 (100) & $5 / 6(83)$ & $8 / 9(89)$ & 81 \\
\hline
\end{tabular}

*www.cdc.gov/tb/programs/evaluation/pdf/National_TB_Objectives_2020_Targets_20160307.pdf.

†Fraction of sputum smear-positive cases in whom the presence (or absence) of close contacts was documented.

¥Fraction of close contacts screened for TB and latent TB infection, relative to the total number of close contacts reported.

§Fraction of close contacts with latent TB infection in whom preventive therapy was commenced.

IFraction of close contacts who completed preventive therapy of those who commenced it; contacts routinely received 6-9 months of isoniazid.

CDC, Centre for Disease Control and Prevention; CXR, chest radiograph; NA, not applicable; PTB, pulmonary tuberculosis; TB, tuberculosis; TST, tuberculin skin test.

A frequently encountered dilemma in close contacts who are TST-positive at baseline is the distinction between recent and past infections, especially in people with previous TB exposure. Although this distinction can be problematic, new global guidance encourages LTBI treatment irrespective of the likely timing of primary infection, ${ }^{21}$ in order to reduce the reservoir of LTBI from which future cases may arise. Both historic and recent studies suggest that this provides a highly effective and cost-efficient strategy to prevent secondary cases and reduce TB case numbers over time, ${ }^{325-27}$ although it remains difficult to consider the effect of possible future reinfection in highly mobile populations. ${ }^{28}$

Guidelines in many countries used to discourage the use of isoniazid preventive therapy in people older than 35 years of age given hepatotoxicity concerns. However, in the absence of excessive alcohol use or known liver disease, the risk of isoniazid-induced hepatotoxicity is mainly elevated in people older than 60 years of age, and the most recent National Institute for Health and Care Excellence guideline (2016) recommends preventive therapy up to the age of 65 years. ${ }^{29}{ }^{30}$ Newer treatment regimens such as weekly isoniazid and rifapentine for 3 months (12 weekly doses), which is now the standard of care in the USA, are generally well tolerated across a wide age range, ${ }^{31}$ but rifapentine access outside the USA is limited, and it is currently not registered for use in Australia. Previous studies reported poor treatment completion rates in healthy adult contacts provided with 6-9 months of isoniazid preventive therapy, ${ }^{32}$ but our study found excellent treatment completion rates when
LTBI treatment was advised by the local clinician. Adherence rates are expected to be even higher if shorter LTBI treatment regimens become more widely available. Our findings demonstrate that the option of CXR follow-up is hardly satisfactory, given low completion rates.

Apart from the opportunity for disease prevention, TB contact tracing also has important active case-finding value, ${ }^{28} 33$ as demonstrated by the four secondary cases identified during this study. In fact, household contact tracing has been identified as one of the most efficient strategies for active case-finding, both in high and low TB incidence settings. ${ }^{33} 34$ LTBI treatment complements active TB case-finding (detection of prevalent case) to break TB transmission chains, and at a population level the combined effect of active TB case-finding and effective LTBI treatment of close contacts is expected to have a major impact on TB incidence and transmission over the long term. ${ }^{35}$ However, clinical variability in the decision to institute LTBI treatment persists among TB physicians in Australia, ${ }^{36}$ including uncertainty about the risk:benefit ratio of LTBI treatment in cases considered to be at low risk of future disease progression. ${ }^{37}$

Study limitations include the relatively small and geographically focused study population, but we believe it provides a good snapshot of the overall management of TB contacts in Western Sydney, which is highly representative of cosmopolitan urban Australia. BCG vaccination may have confounded some TST results, but BCG vaccination or the presence of a BCG scar was not consistently documented in the clinical notes and could not be analysed. During the study period IGRAs were not available 
for routine LTBI evaluation, but these have since been endorsed for this purpose and widespread use should improve the specificity of LTBI detection in BCG-vaccinated individuals. ${ }^{21} 38$ The HIV status of TB contacts was not recorded, which may significantly increase an individual's risk of developing TB if LTBI is detected. ${ }^{39}$ However, HIV testing of TB contacts is not routinely performed given that the prevalence of HIV infection in Western Sydney is very low, even among patients with TB and their families.

In conclusion, we documented good TB contact elicitation and evaluation rates in Western Sydney, falling just short of the CDC targets. However, few contacts with documented LTBI received preventive therapy, except for children $<5$ years of age. This highlights the challenges of wide-scale LTBI treatment in contacts considered to be at low risk of future disease progression, as well as the need to provide clinicians with feasible and minimal risk treatment options, while policy makers require convincing cost:benefit analyses to motivate widescale implementation.

Acknowledgements We thank the nursing and administration staff involved in the management and contact tracing of pulmonary TB cases and their contacts at Parramatta Chest Clinic. We also thank the NSW Mycobacterial Reference Laboratory, especially Professor Vitali Sintchenko and Basel Suliman, for providing the available culture, MIRU-24 and drug susceptibility testing data.

Contributors LP and SKB drafted the proposal with input from all authors. LP, NH, HA and SN helped to collect and analyse the data. LP, SKB and J-GC drafted the initial manuscript with guidance from SC and BJM. All authors helped to refine the manuscript and approved the final version.

Funding The authors have not declared a specific grant for this research from any funding agency in the public, commercial or not-for-profit sectors.

Competing interests None declared.

Patient consent Not required.

Ethics approval Ethics approval was obtained from the Western Sydney LHD Human Research Ethics Committee (AU RED LNR/WMEAD/191).

Provenance and peer review Not commissioned; externally peer reviewed.

Data sharing statement Data available upon request.

Open access This is an open access article distributed in accordance with the Creative Commons Attribution Non Commercial (CC BY-NC 4.0) license, which permits others to distribute, remix, adapt, build upon this work non-commercially, and license their derivative works on different terms, provided the original work is properly cited, appropriate credit is given, any changes made indicated, and the use is non-commercial. See: http://creativecommons.org/licenses/by-nc/4.0/

\section{REFERENCES}

1. World Health Organization. The end TB strategy. Geneva, Switzerland, 2014. Available from: http://www.who.int/tb/strategy/ End_TB_Strategy.pdf?ua=1 [accessed 9 May 2017].

2. Lönnroth K, Migliori GB, Abubakar I, et al. Towards tuberculosis elimination: an action framework for low-incidence countries. Eur Respir J 2015;45:928-52.

3. Rangaka MX, Cavalcante SC, Marais BJ, et al. Controlling the seedbeds of tuberculosis: diagnosis and treatment of tuberculosis infection. Lancet 2015;386:2344-53.

4. World Health Organization. Global strategy and targets for tuberculosis prevention, care and control after 2015. Geneva, Switzerland, 2017. Available from: http://apps.who.int/gb/ebwha/ pdf_files/EB134/B134_12-en.pdf?ua=1 [accessed 2 May 2017].

5. Communicable Diseases Network Australia. The strategic plan for control of tuberculosis in Australia: 2011-2015. Australia, 2012. Available from: http://www.health.gov.au/internet/main/publishing. nsf/Content/cda-cdi3603i.htm [accessed 1 May 2017].
6. Toms $C$, Stapledon $\mathrm{R}$, Coulter $\mathrm{C}$, et al. Tuberculosis notifications in Australia, 2014. Commun Dis Intell Q Rep 2017;41:E247-E63.

7. Toms C, Stapledon R, Waring J, et al. Tuberculosis notifications in Australia, 2012 and 2013. Commun Dis Intell Q Rep 2015;39:E217-35.

8. NSW Health. Tuberculosis New South Wales: 2012-2014. NSW, Australia, 2014. Available from: http://www.health.nsw.gov.au/ Infectious/tuberculosis/Documents/tb-report-2012-14.pdf [accessed 2 April 2018]

9. Zumla A, George A, Sharma V, et al. The WHO 2014 global tuberculosis report--further to go. Lancet Glob Health 2015;3:e10-e12.

10. Epidemiology EMSWSLHD Social and Health Atlas, 2012. Epidemiology, Executive medical services. Available from: https:// www.wslhd.health.nsw.gov.au/SocialHealthAtlas/ [accessed 2 April 2018].

11. NSW Health. Tuberculosis contact tracing [Policy Directive]. NSW, Australia, 2008. Available from: http://www1.health.nsw.gov.au/pds/ Pages/doc.aspx?dn=PD2008 017 [accessed 27 Jun 2017].

12. Department of Health. The role of tuberculosis services in NSW, Australia, 2014. Available from: http://www.health.nsw.gov.au/Infectious/ tuberculosis/Documents/GPCTB-role-of-TB-services.pdf [accessed 21 Jun 2017]

13. Centers for Disease Control and Prevention. Tuberculin skin testing,testing and diagnosis, USA, 2016. Available from: https://www.cdc.gov/tb/ publications/factsheets/testing/skintesting.htm [accessed 2 Jul 2018].

14. World Health Organization. Guidance for national tuberculosis programmes on the management of tuberculosis in children. Geneva, Switzerland, 2014. Available from: http://apps.who.int/ medicinedocs/documents/s21535en/s21535en.pdf [accessed $3 \mathrm{Jul}$ 2017].

15. Centers for Disease Control and Prevention. Latent tuberculosis infection: guide for primary health care providers. GA, USA, 2014 Available from: https://www.cdc.gov/tb/publications/ltbi/diagnosis. htm [accessed 1 Jun 2017].

16. Centre for Epidemiology and Evidence. Notifiable Conditions. Australia: NSW Health, 2017. Available from: http://www.health.nsw. gov.au/epidemiology/Pages/notifiable-conditions.aspx [accessed 1 Jul 2018].

17. Centers for Disease Control and Prevention. National TB program objectives and performance target for 2020. GA, USA, 2015. Available from: https://www.cdc.gov/tb/programs/evaluation/ indicators/default.htm [accessed 1 Jun 2017].

18. Marks SM, Taylor Z, Qualls NL, et al. Outcomes of contact investigations of infectious tuberculosis patients. Am J Respir Crit Care Med 2000;162:2033-8.

19. Cavany SM, Sumner T, Vynnycky E, et al. An evaluation of tuberculosis contact investigations against national standards. Thorax 2017;72:736-45.

20. Perez-Velez CM, Marais BJ. Tuberculosis in children. N Engl J Med Overseas Ed 2012;367:348-61.

21. World Health Organization. Latent tuberculosis infection, updated and consolidated guidelines for programmatic management Geneva, Switzerland, 2018. Available from: http://www.who.int/tb/ publications/2018/latent-tuberculosis-infection/en/ [accessed 1 Jun 2018].

22. Marais BJ, Obihara CC, Warren RM, et al. The burden of childhood tuberculosis: a public health perspective. Int $J$ Tuberc Lung Dis 2005;9:1305-13.

23. Hill PC, Rutherford ME, Audas R, et al. Closing the policy-practice gap in the management of child contacts of tuberculosis cases in developing countries. PLoS Med 2011;8:e1001105.

24. Brigden G, Furin J, Van Gulik C, et al. Getting it right for children: improving tuberculosis treatment access and new treatment options. Expert Rev Anti Infect Ther 2015;13:451-61.

25. Houben RM, Dodd PJ. The global burden of latent tuberculosis infection: a re-estimation using mathematical modelling. PLoS Med 2016;13:e1002152.

26. Patel AR, Campbell JR, Sadatsafavi M, et al. Burden of nonadherence to latent tuberculosis infection drug therapy and the potential cost-effectiveness of adherence interventions in Canada: a simulation study. BMJ Open 2017;7:e015108.

27. Whalen CC. Diagnosis of latent tuberculosis infection: measure for measure. JAMA 2005;293:2785-7.

28. Fox GJ, Dobler CC, Marais BJ, et al. Preventive therapy for latent tuberculosis infection-the promise and the challenges. Int $J$ Infect Dis 2017;56:68-76.

29. National Institute for Health and Clinical Excellence (NICE). Tuberculosis (NICE guideline 33). London, 2016. Available from: https://www.nice.org.uk/guidance/ng33 [accessed 19 Jul 2018] 
30. Hosford JD, von Fricken ME, Lauzardo M et al. Hepatotoxicity from antituberculous therapy in the elderly: a systematic review. Tuberculosis 2015;95:112-22.

31. Sterling TR, Villarino ME, Borisov AS, et al. Three months of rifapentine and isoniazid for latent tuberculosis infection. $N$ Engl J Med Overseas Ed 2011;365:2155-66.

32. Ziakas PD, Mylonakis E. 4 months of rifampin compared with 9 months of isoniazid for the management of latent tuberculosis infection: a meta-analysis and cost-effectiveness study that focuses on compliance and liver toxicity. Clin Infect Dis 2009;49:1883-9.

33. Fox GJ, Nhung NV, Sy DN, et al. Household-Contact Investigation for Detection of Tuberculosis in Vietnam. N Engl J Med Overseas Ed 2018;378:221-9.

34. Fox GJ, Barry SE, Britton WJ, et al. Contact investigation for tuberculosis: a systematic review and meta-analysis. Eur Respir J 2013;41:140-56.
35. Ragonnet R, Trauer JM, McBryde ES, et al. Is IPT more effective in high-burden settings? Modelling the effect of tuberculosis incidence on IPT impact. Int J Tuberc Lung Dis 2017;21:60-6.

36. Denholm JT, McBryde ES. Management of latent tuberculosis infections in Australia and New Zealand: a review of current practice. Tuberc Res Treat 2010:1-5.

37. Dobler CC, Bosnic-Anticevich S, Armour CL. Physicians' perspectives on communication and decision making in clinical encounters for treatment of latent tuberculosis infection. ERJ Open Res 2018;4:00146-2017.

38. Bibbins-Domingo K, Grossman DC, Curry SJ, et al. Screening for Latent Tuberculosis Infection in Adults: US preventive services task force recommendation statement. JAMA 2016;316:962-9.

39. World Health Organization. Global tuberculosis report 2017. Geneva, Switzerland, 2017. Available from: http://www.who.int/tb/ publications/global_report/en/ [accessed 6 Jun 2018]. 